\title{
Small Sample Data Method Research on Reliability Modeling of Direct Reusing Electromechanical Servo System
}

\author{
MA Wenbin ${ }^{\mathrm{a}}$, Liu Jianhua and ZhouZiqiang \\ Changshu Institute of Technology, JiangSu china \\ mawb_suzhou@163.com
}

\begin{abstract}
Keywords: sample data method; Direct reutilization of servo motor; reliability modeling
\end{abstract}
\begin{abstract}
Servo motor is one of the high value-added mechanical and electrical products, The servomotor disassembled from the waste equipment can be reused after the performance index is verified. But there is always a doubt about its reliability in the process of reuse. Based on the Bayes reliability theory, this paper establishes a small sample reused reliability model for the direct reuse of the servo motor. And the identification method of model parameters is given, and the experimental conditions are given. According to the theory, the reliability model based on the data is established according to the theory.
\end{abstract}

\section{Introduction}

With the progress of society, the concept of green manufacturing and intelligent manufacturing has been implemented in the whole industrial system. With the upgrading of industry, a lot of waste electrical and mechanical equipment is facing scrapping, resulting in great economic losses and environmental losses. Dismantling valuable electromechanical components from waste electrical and mechanical equipment and recycling them after performance testing become a way of comprehensive recycling of mechanical and electrical products. Organization

The recovery of the servo motor by research is not much, in reliability modeling, Bayesian reliability theory established by [1] engineering machinery reliability model based on small sample data by Xuzhou Engineering Research Institute of Zhang Hong and Zhang Haijun; Zhang Genbao of Chongqing University based on FAM method was established for complex electromechanical product reliability and fault diagnosis model of [2]; Beijing University of Technology Fan Jinwei established the [3] hydraulic system reliability model of CNC grinding machine;

The reliability modeling of small sample based on Bayesian method can effectively use prior information to solve the problem of small sample data modeling.

\section{Small Samples Bayes Reliability Model}

Suppose the product of the experiment has $r$ values in the prescribed end time T. After fault interval time sorting: $t_{1} \leq t_{2} \leq \cdots \leq t_{r} \leq T$, fault probability $p_{i}=F\left(t_{i}\right), i=1,2, \cdots, r$; For $p_{i}=F\left(t_{i}\right), i=1,2, \cdots, r$.

$$
0 \leq p_{i} \leq 1-\left(1-p_{r}\right)^{t_{i} / t_{r}}
$$

The case of a small number of failures is the case of a small number of failures. The failure probability is not very large before the end of the time truncation experiment. Set $\mathrm{T}$ for a given time, the $\mathrm{T}$ time failure probability is $\mathrm{p}$. The upper limit of $\mathrm{p}$ is $p_{u} \cdot p_{u}$ can be obtained from the experience of the past. The prior distribution function required by the Bayes estimation is further obtained[4].It is known from the above discussion $0 \leq p \leq p_{u}$, The uniform distribution on the prior distribution is considered as $\left[0, p_{u}\right]$ : 


$$
\Pi(p)=\left\{\begin{array}{l}
\frac{1}{p_{u}}, 0 \leq p \leq p_{u} \\
0
\end{array}\right.
$$

It is known from the formula (1), for $p_{i}=1-(1-p)^{\frac{t_{i}}{T}}$, So the prior distribution is as follows:

$$
\Pi_{i}\left(p_{i}\right)=\Pi(p) \frac{d p}{d p_{i}}=\frac{T}{p_{u} t_{i}}\left(1-p_{i}\right)^{\frac{T}{t_{i}}-1}
$$

During t moment it's i times faults happened. So the likelihood function of the fault probability is two term distribution.

$$
L\left(p_{i}\right)=\frac{n !}{i !(n-i) !} p_{i}\left(1-p_{i}\right)^{n-i}
$$

$\mathrm{i}$ is the number of failures that have occurred, $1 \leq i \leq r$;n is The sum of the number of failures and the number of the failed equipment. According to (3) and (4), $p_{i}$ can be described as:

$$
\Pi\left(p_{i} \mid t_{i}\right)=\frac{\Pi\left(p_{i}\right) L\left(p_{i}\right)}{\int_{0}^{p_{i u}} \Pi_{i}\left(p_{i}\right) L\left(p_{i}\right) d p_{i}}=\frac{p_{i}\left(1-p_{i}\right)^{\frac{T_{i}}{t_{i}}+n-i-1}}{\int_{0}^{p_{i u}} p_{i}\left(1-p_{i}\right)^{\frac{T}{t_{i}}+n-i-1}}
$$

$p_{\text {iu }}$ is the upper limit of the number of failures. Loss function is the squared loss. Bayes estimate:

$$
\hat{p}_{i}=\int_{0}^{p_{i u t}} p_{i} \Pi_{i}\left(p_{i} \mid t_{i}\right) d p_{i}
$$

According to experiment $\left(t_{i}, \widehat{p}_{i}\right)$ data, Parameter estimate value can be get of reliability model from least square method. Setting $\mathrm{R}(\mathrm{t})$ is reliability function. Constraint criterion of least square method:

$$
Q=\sum_{i=1}^{r} \omega_{i}\left[1-\hat{p}_{i}-R\left(t_{i}\right)\right]^{2}
$$

$\mathrm{Q}$ is the Minimum objective function. $\omega_{i}$ is the weight coefficient. $\omega_{i}$ can be get from:

$$
\omega_{i}=\sum_{j=1}^{i} t_{j} /\left[\sum_{j=1}^{r} t_{j}+(n-r) T\right]
$$

According to (7), when $\mathrm{Q}$ is the minimum, parameter estimation can be get[5].

\section{Example Analysis}

In order to estimate one kind of servo system reliability,10 servo equipment by random selection are checkout. The time truncation is adopted in the experiment. According to the actual situation, the scheduled truncation time is 3000 hours. In the experiment of industrial assessment, assessment of product failures will be deal with the standard of failure. A fault mark that affects the completion of a product task is a small fault, failures that do not affect the completion of a product task are not recorded. Stop the experiment and organize the fault data at the end of the truncation, 6 failures ware founded and the alarm fault caused by load is up to 2 times, heating alarm 3 times due to high speed and frequent reversal, one time fault by other situation. The interval between each fault is shown in Table 1.The time data in the table are the intervals between the two adjacent faults after processing. 
Evaluation of product reliability by using small sample Bayes reliability model, the upper limit of the failure probability of the product at $\mathrm{T}$ time is set to 0.5 based on the experience of use.

Table 1 a fault data table for a servo system

\begin{tabular}{|l|l|}
\hline Number of failures & Fault interval time/h \\
\hline 6 & $134.23,435.23,498.11,573.21,1131.23,1531.64$ \\
\hline
\end{tabular}

According to (4),

$$
p_{1 u}=1-\left(1-p_{u}\right)^{t / 1}=1-(1-0.5)^{134.223 / 3000}=0.0305
$$

Similarly, $p_{1 u}-p_{6 u}$ can be get as shown in Table 2.According to (5)and(6), $\hat{p}_{1}-\hat{p}_{6}$ can be get.

Table 2 the value of $p_{i u}$

\begin{tabular}{|c|c|c|c|c|c|}
\hline$p_{1 u}$ & $p_{2 u}$ & $p_{3 u}$ & $p_{4 u}$ & $p_{5 u}$ & $p_{6 u}$ \\
\hline 0.0305 & 0.0957 & 0.1087 & 0.1240 & 0.23 & 0.298 \\
\hline
\end{tabular}

Table 3 the value of $\hat{p}_{i}$

\begin{tabular}{|c|c|c|c|c|c|}
\hline$\hat{p}_{1}$ & $\hat{p}_{2}$ & $\hat{p}_{3}$ & $\hat{p}_{4}$ & $\hat{p}_{5}$ & $\hat{p}_{6}$ \\
\hline 0.0125 & 0.0751 & 0.0897 & 0.107 & 0.211 & 0.279 \\
\hline
\end{tabular}

According to (8), $\omega_{1}-\omega_{6}$ can be get.

Table 4 the value of $\omega_{i}$

\begin{tabular}{|c|c|c|c|c|c|}
\hline$\omega_{1}$ & $\omega_{2}$ & $\omega_{3}$ & $\omega_{4}$ & $\omega_{5}$ & $\omega_{6}$ \\
\hline 0.0045 & 0.035 & 0.0325 & 0.043 & 0.097 & 0.128 \\
\hline
\end{tabular}

\section{Reliability Model Test}

The Weibull distribution model is widely used in reliability evaluation and verification test of mechanical, electronic and aerospace products in reliability analysis. For the collected fault data, the two parameter Weibull test is carried out by using MATLAB, the result shown $\mathrm{P}>0.05$.It can be considered that the failure data of the product is subject to the Weibull part.

\section{Algorithm Optimization}

Using the least squares constraint criterion to set the target function $Q$, optimize $\alpha, \beta$ value of the model, which can make Q up to minimum. The optimize step as following:

(1) the $\omega_{i}, \hat{p}_{i}, t_{i}$ value should be inputted to calculate system;

(2) initialize the parameter of calculate system;

(3) Because of two parameter $\alpha, \beta$,each calculate unit have two part, which means the calculate dimension $\mathrm{D}$ is 2 , and the number of calculate unit is 100 .Maximum evolutionary value $\mathrm{G}$ is 150 ; Variation factor $\mathrm{V}$ is 0.5 ; Cross probability CR is 0.9 ;

(4) Random select one of $\alpha, \beta$, and begin optimize;

(5) Mutation and cross operation for each individual unit;

(6) Taking $\mathrm{Q}$ as the objective function, select operations for the next generation;

(7) Realized through practical programming, when $\alpha=10324.33, \beta=1.3713, Q_{\min }=13.712$.

\section{Reliability Model}

According to the two parameter optimization algorithm, the value of the reliability model of the product can be obtained. On this basis, the probability density function and distribution function of the fault interval time of the product can be obtained, as (10)-(13): 


$$
f(t)=\frac{\beta}{\alpha}\left(\frac{t}{\alpha}\right)^{\beta-1} \exp \left(-\left(\frac{t}{\alpha}\right)^{\beta}\right)
$$

Input $\alpha=10324.33, \beta=1.3713$ to (10):

$$
\begin{aligned}
& f(t)=\frac{1.3713}{10324.33}\left(\frac{t}{10324.33}\right)^{0.3713} \times \exp \left(-\left(\frac{t}{10324.33}\right)^{1.3713}\right) \\
& F(t)=1-\exp \left(-\left(\frac{t}{10324.33}\right)^{1.3713}\right)
\end{aligned}
$$

So reliability model as (13) describe:

$$
R(t)=\exp \left(-\left(\frac{t}{10324.33}\right)^{1.3713}\right)
$$

\section{Conclusion}

In the reliability research of electromechanical servo system, small sample is common. Based on Bayes reliability theory, a reliability model of electromechanical system is established, and the parameter estimation method of reliability model is given, and the reliability model in the example is obtained.

\section{References}

[1] Zhang Hong, Zhang Haijun, Reliability modeling method of whole machine product based on small sample data.,Construction Machinery,10 (2017) 37-40.

[2] Zhang Genbao,WangLin, Reliability modeling and fault diagnosing method of complex electromechanical products based on meta-action decomposition , Journal of Chongqing University, 40(8), 2017.9-18

[3] Fan Jinwei, Zhang Lanqing, Building Reliability Model and Evaluating the Hydraulic System in CNC Grinding Machine, Machinery Design and Manufacture,8(8)2017.90-92

[4] Li D Y,Zhang G B.Assembly reliability modeling technology based on meta-action, Procedia Cirp,2015(27):207-2015

[5] DaiYi,Zhou Yifei. Distribution of time between failures of machining center based on type I censored data,Reliability Engineering and System Safety,79(3),2003:375-377 